\title{
Does Unit-Tested Code Crash? A Case Study of Eclipse
}

\author{
Efstathia Chioteli* \\ Ioannis Batas* \\ Diomidis Spinellis* \\ t8150148@aueb.gr \\ t8150090@aueb.gr \\ dds@aueb.gr \\ Department of Management Science and Technology \\ Athens University of Economics and Business \\ Athens, Greece
}

\begin{abstract}
Context: Software development projects increasingly adopt unit testing as a way to identify and correct program faults early in the construction process. Code that is unit tested should therefore have fewer failures associated with it.

Objective: Compare the number of field failures arising in unit tested code against those arising in code that has not been unit tested.

Method: We retrieved 2083979 crash incident reports associated with the Eclipse integrated development environment project, and processed them to obtain a set of 126026 unique program failure stack traces associated with a specific popular release. We then run the JaCoCo code test coverage analysis on the same release, obtaining results on the line, instruction, and branch-level coverage of 216392 methods. We also extracted from the source code the classes that are linked to a corresponding test class so as to limit test code coverage results to 1267 classes with actual tests. Finally, we correlated unit tests with failures at the level of 9523 failing tested methods.

Results: Unit-tested code does not appear to be associated with fewer failures.

Conclusion: Unit testing on its own may not be a sufficient method for preventing program failures.

\section{CCS CONCEPTS}

- Software and its engineering $\rightarrow$ Software testing and debugging.

\section{KEYWORDS}

Unit-testing, crash incident reports, code coverage, stack traces, software reliability

${ }^{*}$ Chioteli collected the unit testing results and performed the qualitative analysis. Batas performed the quantitative analysis. All authors contributed equally to the paper's writing.
\end{abstract}

Unpublished working draft. Not for distribution.

2020-03-03 02:22. Page 1 of 1-12.
ACM Reference Format:

Efstathia Chioteli, Ioannis Batas, and Diomidis Spinellis. 2020. Does UnitTested Code Crash? A Case Study of Eclipse. In MSR '20: 17th International Conference on Mining Software Repositories, May 25-26, 2020, Yongsan-gu, Seoul, South Korea. ACM, New York, NY, USA, 12 pages. https://doi.org/10. $1145 / 1122445.1122456$

\section{INTRODUCTION}

The rising size and complexity of software multiply the demands put on adequate software testing [25]. Consequently, software development projects increasingly adopt unit testing [6] or even test-driven development [5] as a way to identify and correct program faults early in the construction process. However, the development of testing code does not come for free. Researchers have identified that one of the key reasons for the limited adoption of test-driven development is the increased development time [7]. It is therefore natural to wonder whether the investment in testing a program's code pays back through fewer faults or failures.

One can investigate the relationship between the software's production code and its tests by utilizing heuristics or code coverage analysis [39]. Heuristics are based on conventions associated with the development of unit test code; for example that a test class is named after the class it tests (e.g. Employer), followed by the Test suffix (i.e. EmployerTest). Code coverage analysis is a process that provides metrics indicating to what extent code has been executedunder various control flow measures [4]. The corresponding metrics can be efficiently obtained through diverse tools [14, 36]. Then, the process for determining test coverage involves running the software's test suite, and obtaining code coverage metrics, which in this case indicate code that (probably) is or (definitely) is not tested.

To examine how test code coverage relates to software quality, numerous methods can be employed. One can look at corrected faults and see whether the corresponding code was tested or not [24, 28]. In addition, faults can be deliberately introduced by mutating the code [20] in order to look at how test code coverage relates to test suite effectiveness $[12,17]$. Alternatively, one can artificially vary test coverage to see its effect on exposing known faults [25]. Finally, one could look at software failures rather than faults and correlate these with test code coverage.

In this study we investigate the relationship between unit testing and failures by examining the usage of unit testing on code that is associated with failures in the field. We do this in three conceptual 
steps. First, we run software tests under code coverage analysis to determine which methods have been unit tested and to what extent. We triangulate these results with heuristics regarding the naming of classes for which unit test code actually exists. Then, we analyze the stack traces associated with software failure reports to determine which methods were associated with a specific failure. Finally, we combine the two result sets and analyze how unit-tested methods relate to observed failures.

We frame our investigation in this context through the following research questions.

RQ1 How does the testing of methods relate to observed failures? RQ2 Why do unit-tested methods fail?

A finding of fewer failures associated with tested code would support the theory that unit testing is effective in improving software reliability. Failing to see such a relationship would mean that further research is required in the areas of unit test effectiveness (why were specific faults not caught by unit tests) and test coverage analysis (how can coverage criteria be improved to expose untested faults).

The main contributions of our study are the following:

- a method for investigating the effectiveness of unit testing,

- an empirical evaluation between unit test coverage and failure reports, and

- an open science data set and replication package providing empirical backing and replicability for our findings.

In the following sections we describe the methods we used (Section 2), present our quantitative and qualitative results (Section 3), discuss their implications (Section 4), examine the threats to the validity of our findings (Section 5), outline related work in this area (Section 6), and conclude with a summary of our findings and their implications (Section 7).

\section{METHODS}

We based our study on the popular Eclipse open source integrated development environment [11]. In brief, to answer our research questions we obtained data regarding failures of the Eclipse IDE, we determined the most popular software version associated with the failures, we built this specific software version, we run the provided tests under a code coverage analysis tool, we combined the results with heuristics regarding the naming of test code, we joined the analyzed software failures with the corresponding code coverage analysis results, and we analyzed the results through statistics and a qualitative study. Following published recommendations [16], the code and data associated with this endeavor (AERI JSON data, code coverage analysis, stack traces analyzed, analysis scripts, and combined results) are openly available online. ${ }^{1}$

In our presentation we use the following terms as defined in the systems and software engineering vocabulary standard [18].

Error "Human action that produces an incorrect result."

Fault "Incorrect step, process, or data definition in a computer program"; "defect in a system or a representation of a system that if executed/activated could potentially result in an error."

\footnotetext{
${ }^{1} 10.5281 /$ zenodo. 3610822
}

Failure "Termination of the ability of a system to perform a required function or its inability to perform within previously specified limits; an externally visible deviation from the system's specification."

According to these definitions, a programmer error may result in a fault in the code. This may in turn cause a failure in the program's operation, which may manifest itself as e.g. incorrect output, a program freeze, or an abnormal program termination; the last one often accompanied by a diagnostic report, such as a stack trace.

\subsection{Data Provenance and Overview}

We conducted our research on a dataset of anonymized diagnostic failure reports communicated to the Eclipse developers through the IDE's Automated Error Reporting (AERI) system [3]. The AERI system is installed by default on the Eclipse IDE to aid support and bug resolution. Its back-end collects incidents, which contain data regarding a particular instance of an uncaught exception. It analyses them and aggregates similar ones into problems. The specific data set we used was generated on 2018-02-17 and contains data collected over the period 2016-03-13 to 2016-12-13. The dataset contains a file with the complete collected data and two extracts in CSV format containing a subset of fields and aggregate data. We based our study on the set titled "All Incidents", which consists of 2083979 crash reports provided in the form of JSON files. ${ }^{2}$

As subset of an AERI report in JSON format appears in Listing 1 . The data attributes that are interesting for the purpose of our research are the following:

- EclipseProduct, the product associated with the Eclipse project,

- BuildId, the version of the Eclipse source code, and

- Stacktrace, the incident's stack trace. Each stack trace consists of successive stack frames and their details (class, method, line).

To combine incident reports with the associated source code, compiled code, and test data, we decided to focus our study on a specific version of Eclipse. We therefore analyzed the AERI incident reports to find the Eclipse release associated with the highest number. This would allow us to obtain a large dataset for statistical analysis. Given that production releases are widely distributed, numerous failures associated with a release are a sign of the release's popularity, rather than its inherent instability. An overview of the incidents associated with each release can be found in the AERI incidents analysis report [3, p. 18]. The data corresponding to the selected version consist of 126026 incident files that have EclipseProduct equal to org. eclipse.epp. package. java.product and BuildID equal to 4.5.2.M20160212-1500.

\subsection{Generation of Test Code Coverage Data}

To create code coverage data associated with tests we needed to obtain the source code, compile it, and perform code coverage analysis while running the tests.

We accessed the Eclipse source code through the Eclipse Platform Releng project, ${ }^{3}$ which provides instructions for building the Eclipse Platform using preferred technologies identified as part

\footnotetext{
${ }^{2}$ http://software-data.org/datasets/aeri-stacktraces/downloads/incidents_full.tar.bz2 ${ }^{3}$ https://wiki.eclipse.org/Platform-releng/Platform_Build
} 


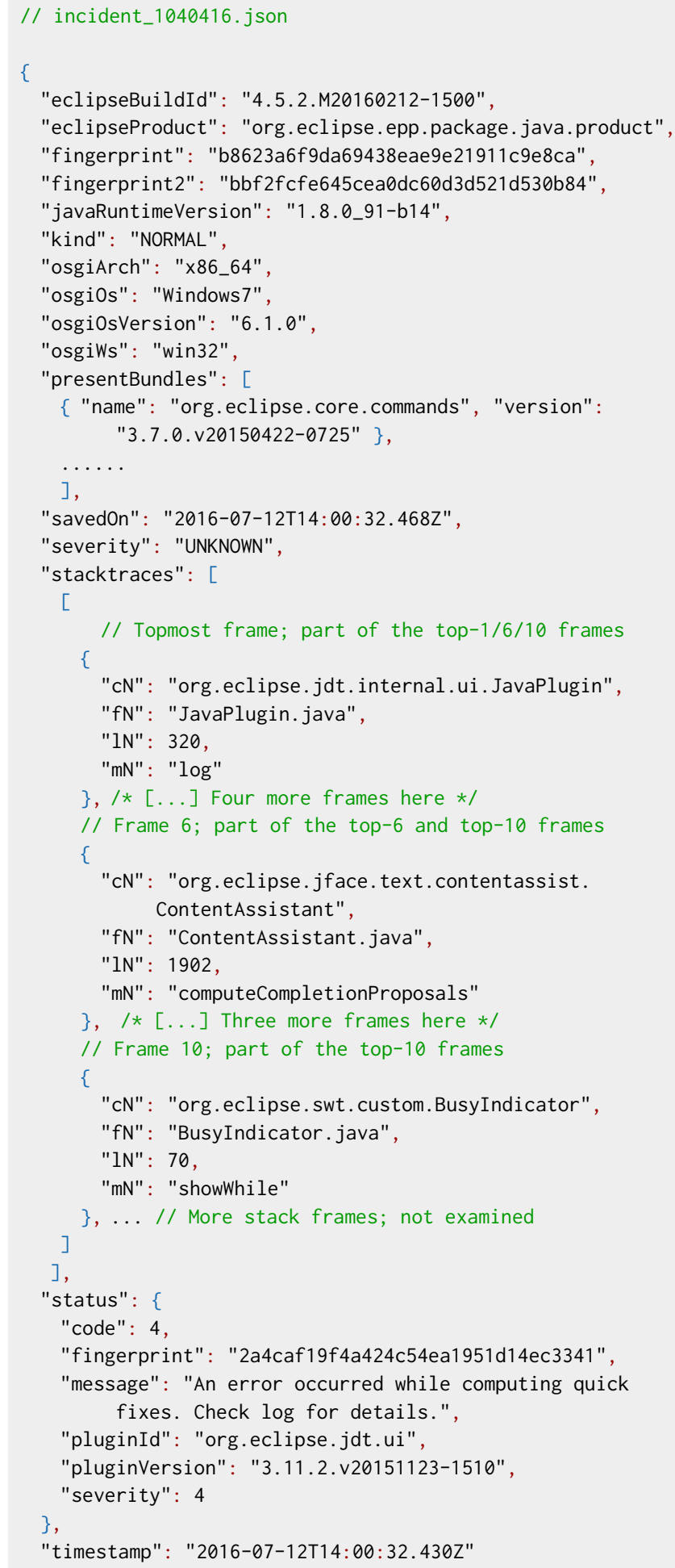

Listing 1: AERI incident data extract of the Eclipse Common Build Infrastructure (CBI) initiative. This combines infrastructure, technologies, and practices for building Eclipse software. To ensure that test coverage results would be coeval with the corresponding incident reports, we retrieved the source code version of Eclipse corresponding to the one whose stack traces we chose to analyze.

To obtain data regarding Eclipse's test coverage, we used the JaCoCo Code Coverage [14] system, which is an open-source toolkit for measuring and reporting Java code coverage. It reports instruction, branch, line, method, class, package, and complexity coverage. Instruction is the smallest unit $\mathrm{JaCoCo}$ counts and is associated with single Java byte code instructions. Branch coverage reports on taken and non-taken branches. Cyclomatic complexity coverage reports the ratio of the executed cyclomatic complexity [27] graph paths over the total cyclomatic complexity number. Under line coverage, a source code line is considered to be covered if at least one instruction associated with the line has been executed. Finally, coverage of larger aggregates is reported on the basis of at executing at least a single instruction. For the purposes of this study we focused on the most fine grained coverage metrics, namely line, instruction, and branch coverage.

Eclipse is a multi-module project, which hinders the derivation of code coverage reports, because the JaCoCo Maven goals used to work on single modules only. For that reason, we used the new "Maven Multi-Module Build" feature, ${ }^{4}$ which implements a Maven goal called "jacoco:report-aggregate". This aggregates coverage data across Maven modules.

To apply this feature, we first added the JaCoCo plugin and profile in the Maven parent pom.xml file, and then we created a separate project where we:

- configured the report-aggregate goal,

- added as dependencies with scopecompile the projects containing the actual code and with scopetest the projects containing the tests and the .exec-suffixed data.

Owing to the size and complexity of Eclipse, the process of compiling, testing, and obtaining code coverage results was far from trivial.

First, due to the fact that we run JaCoCo on an old (by about three years) release of Eclipse, it was difficult to make the code compile. The release comprised some repositories which had become archived at the time we attempted to build it, and therefore the specified path could not found. For example, in one case Maven terminated with an internal error reporting that it had failed to load to the repository eclipse-p2-repo from location http://downl oad.eclipse.org/eclipse/updates/4.5-M-builds. To address this issue we replaced these repositories with newer ones.

Second, three tests remained stuck for more than one hour. One reason we might think this was happening was because the test was repeatedly trying to find a specific file. In the end we had to manually remove the offending tests in order to proceed with testing and code coverage analysis process.

Third, there were tests that needed specific configurations and data to run. Again, we had to skip those tests in order to allow the remaining ones to run.

$\overline{{ }^{4} \text { https://github.com/jacoco/jacoco/wiki/MavenMultiModule }}$ 
These three problems resulted in a very time-consuming process, because every time a run failed, we had to fix and restart it to find the next missing repository or stuck test. The average time of each run was about three hours. In total we spent around three months fine-tuning the compilation process and the tests, until we were able to compile the project from source and run most of the tests to obtain test code coverage results.

\subsection{Determination of Unit Test Classes}

Code coverage data can be a notoriously fallacious measure of test quality [35]. Because good quality tests are associated with high code coverage and the absence of tests with low code coverage, many mistakenly think that high code coverage implies good quality testing. In fact, high code coverage can be achieved by having some code executed without testing its correct behavior. As an example the code for setting up a test case can invoke some class constructors, from the same or from another class, without however checking that the corresponding objects are correctly constructed.

To alleviate false positives regarding the existence of tests that would result from naively analyzing code coverage reports (class $A$ got executed, therefore it is tested), we combined method-level code coverage data with data regarding the existence of classes containing unit tests.

Specifically, we found the classes of the source code that are relevant to a test class with the following procedure. A common unit test naming practice is to add the word 'Tests' in the end of the class name. However, that is not the case for the test methods (whose name may be quite different) and so we focused only on finding the unit-tests on class level.

Through manual examination of the source code we determined that Eclipse's source code test files (classes) are usually named as follows.

(1) ClassName'Tests

(2) ClassNameTest

(3) TestClassName

(4) Test_PackageName_ClassName

(5) ClassName Tester

Another common unit test practice is to place test files under the tests/ folder with similar path as the class that they test. For example:

Bundle Class: eclipse.platform.ui/bundles/org.ecl ipse.jface.databinding/src/org/eclipse/jface/d atabinding/swt/WidgetProperties. java

Test Class: eclipse.platform.ui/tests/org.eclipse. jface.tests.databinding/src/org/eclipse/jface/ tests/databinding/swt/WidgetPropertiesTest. jav a

In the example above, the path is exactly the same apart from the additional 'tests/' folders. However, there were cases on the Eclipse source code, where the test of the class was not placed in the exact path, but we also matched these files since the test class was related to the bundle class. For example:

Bundle Class: eclipse.platform.ui/bundles/org.ecl ipse.jface/src/org/eclipse/jface/preference/Bo oleanFieldEditor. java
Test Class: eclipse.platform.ui/tests/org.eclipse. ui.tests/EclipseJFaceTests/org/eclipse/jface/t ests/preferences/BooleanFieldEditorTest. java

We wrote a script to find the classes that have a corresponding unit test file by devising through successive experiments and implementing the following heuristics. For each sub-module we generated two sets: one of files containing in their name the word "test" and its complement. We then matched the two sets, by removing the word "test" from the filename and also by traversing the associated paths from the right to the left. In cases where this method failed, we matched files based on the number of same words in each path. In all cases but one we had one or more test files match a single class file. The cases where this relationship did not hold concerned the separate implementations of SWT for the Cocoa, GKT, and Win32 back-ends, which all shared the same test class eclipse.platform.swt/tests/org. eclipse.swt. test s/JUnitTests/org/eclipse/swt/tests/junit/Test_org_ec lipse_swt_widgets_Text.java. Also in another 15 cases we had the name of multiple test files correspond to multiple classes; we paired these tests with the corresponding classes through manual inspection. Through this procedure we matched 1308 test files with 1267 classes.

In addition, for each bundle class and each test class we counted the number of lines so as to see how well a class is tested (test density).

\subsection{Data Synthesis}

As a next step we combined the data elements derived in the preceding steps as follows.

(1) Process the incident files of the dataset, extracting all methods from the stack traces together with their order of appearance. If a method appeared twice in the stack trace we kept only the very first appearance to avoid duplications.

(2) Process the XML file generated by the JaCoCo coverage report (Section 2.2), extracting all methods together with their code coverage data.

(3) Process the unit test classes file and their line density generated with the method explained in Section 2.3.

(4) Join the common methods of the three preceding lists into a new list containing the combined fields.

The resulting output and its description are provided in the paper's replication package.

\subsection{Preliminary Quantitative Analysis}

Overall, JaCoCo tallied 31181 Eclipse source code classes of which the 10513 were covered (34\%). At the method level, out of 216392 methods the 71238 (33\%) were reported as covered. In addition, JaCoCo reported that:

- 1960447 instructions out of 5788907 were covered (34\%).

- 217622 branches out of 686481 were covered (32\%), and

- 481625 of lines out of 1413785 were covered (34\%),

Based on the small variation of the above figures, we decided to base our analysis on test code line coverage, which is the most frequently used method. 

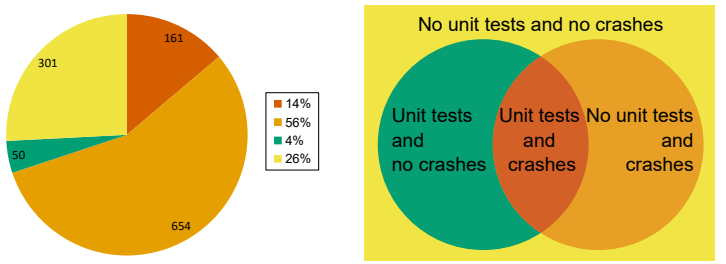

Figure 1: Relationship between test code coverage at the level methods and existence of unit tests at the level of classes. The pie areas correspond to the colored areas of the Venn diagram depicted on the right.

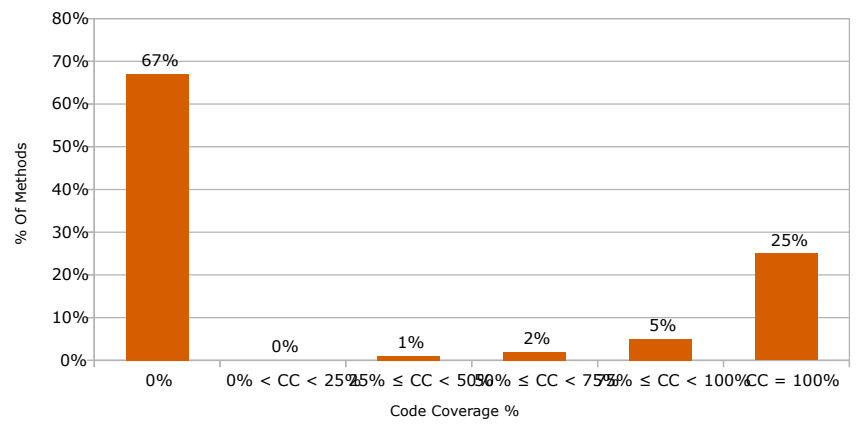

Figure 2: Relationship between code coverage and methods of JaCoCo report.

Given the variation of code coverage within a method's body, we also examined coverage of methods in terms of lines. Interestingly, $25 \%$ of the methods are fully covered, out the $33 \%$ that are covered in total (Figure 2).

In parallel with the JaCoCo code coverage, we found that 1267 classes belong to a class with unit-test code (Section 2.3). The small number of unit-tested classes against the total number of Eclipse's classes can be partly justified by the fact that abstract classes (about two thousand) and interfaces (about six thousand) typically do not have methods to test. The total lines of these classes are 683540 and the total lines of the unit-tested classes is 454938 , giving a test code line density ratio of $67 \%$ and a median test code line density across methods of $71 \%$.

We see that only $12 \%(1267 / 10513)$ of the JaCoCo covered classes belong to unit-tested classes. This small number is justified by the fact that JaCoCo shows which code was executed (or not) when running tests. The coverage does not necessarily mean that a given class was tested, because its code might have been called from other code.

The position of faulty methods in a stack trace was examined by Schroter and his colleagues [34]. They studied 2321 bugs from the Eclipse project, and examined where defects were located in stack traces as defined by the corresponding fix. Their research showed that $40 \%$ of bugs were fixed in the very first frame, $80 \%$ of bugs were fixed within the top- 6 stack frames, and $90 \%$ of bugs were fixed within the top-10 stack frames. We correspondingly grouped and matched methods appearing in stack traces into three groups of methods: those that have appeared at least once in the very first, in the top-6, and in the top-10 stack frames.

2020-03-03 02:22. Page 5 of 1-12.
Table 1: Code Coverage of Methods with Class Unit Tests

\begin{tabular}{lrrrrrr}
\hline & \multicolumn{4}{c}{ Class Unit Test } & \multicolumn{2}{c}{ Total } \\
Covered & \multicolumn{2}{c}{ No } & \multicolumn{2}{c}{ Yes } & \multicolumn{2}{c}{} \\
\hline No & 3115 & $(32.7 \%)$ & 366 & $(3.8 \%)$ & 3481 & $(36.6 \%)$ \\
Yes & 4890 & $(51.3 \%)$ & 1152 & $(12.1 \%)$ & 6042 & $(63.4 \%)$ \\
\hline Total & 8005 & $(84.1 \%)$ & 1518 & $(15.9 \%)$ & 9523 &
\end{tabular}

Following the methods we described in Section 2.4, we matched 14902 crash methods with their test coverage details. Since faulty methods in a stack trace appear mostly, within the top-10 stack frames [34], we excluded the methods that appeared after the 10th stack frame and kept 9523 crash methods.

Of those 9523 crash methods, the 6042 (63\%) are covered according to JaCoCo (instruction\% $>0$ ). In terms of branches they are covered by $55 \%$, in terms of instructions by $58 \%$ and in terms of lines, by 59\%. Also, 1518 (16\%) methods out of 9523 belong to a class with unit test, with average code coverage line density of $71 \%$. The number of methods that belong to a class with unit test and have also non-zero code coverage percentage, is 1152 .

We decided to focus only on the methods appearing in the stack trace's top position, because a) the method at the stack's top, as the one where the exception occurred, is certainly implicated in the crash, even if it may not be the crash's root cause; and b) $40 \%$ of bugs are fixed in the very first frame [34]. The number of crash methods that satisfy this criterion, is 1166 methods out of the 9523 $(12 \%)$.

Drilling further in the association between code coverage and crashes, we examined the relationship between the covered methods, methods of unit-tested classes, and methods of the topmost stack frame. Among 1166 methods associated with failures, test code coverage and the existence of unit tests within the class are related as depicted in Figure 1 and summarized in Table $1 .^{5}$ The numbers we obtained indicate that code coverage on its own cannot be used as a reliable indicator for determining the existence of unit tests. Consequently, we decided to consider as unit tested methods those whose code is covered during testing and whose class has a corresponding one with unit tests.

\subsection{Preliminary Qualitative Analysis}

We conducted a qualitative study of our data so as to gain a better insight of the crashes, the tests, and the code coverage results.

As expected, in the JaCoCo results found methods with code coverage higher than 0 but no tests for their methods. The reason for this is because JaCoCo in common with other code coverage tools shows which instructions, lines, or branches of the code were (or were not) executed when running the tests. This however does not mean that a given method was tested, because its code might have been called by another method's test. Below we outline specific cases of test coverage we related with the existence of actual test code, as outlined in Section 2.3.

1. Methods of classes with unit tests and zero code coverage. There are methods with zero code coverage percentage (according to the $\mathrm{JaCoCo}$ results) that belong to a class that is associated with a test

${ }^{5}$ Also available in the "Metrics" sheet of the paper's replication package. 
class found through the heuristics outlined in Section 2.3. Since it does not make much sense to have unit tests that are not executed, we investigated this further to see why that happens. The main reason seems to be wrong results from JaCoCo, which skipped some tests that were not running due to configuration settings. For example, most of the tests of the module rt.equinox.p2 did not run successfully and so most of its classes and methods got a zero coverage percentage, although they had unit tests. Specifically, the class org.eclipse.equinox. internal.p2.metadata.reposi tory.CompositeMetadataRepository is related to the unit-test class org.eclipse.equinox.p2. tests.metadata. repositor y.CompositeMetadataRepositoryTest which tests some of its methods, but JaCoCo indicated zero coverage for its methods such as the method addchild. Interestingly, this method also appeared on the topmost stack frame of 50 incident reports.

Another case occurs when the test class contains tests for a subset of the class's methods and none of the other unit tests or unit-tested methods execute some methods. This results in JaCoCo indicating no coverage for some methods. Thus the presence of unit tests for a class is no guarantee for unit tests for all the class's methods. We found for example this to be the case in the method doSetValue(Objectsource, Objectvalue) of the class org.e clipse.core. internal. resources. Workspace.

Despite its intuitive justification, this is not a common phenomenon: only 336 methods of those listed in incident stack traces out a total of 9523 belong to this category.

2. Methods of classes without unit tests and non-zero code coverage. This category comprises methods with a non-zero coverage percentage that do not belong to any of the classes with unit test found through the procedure described in section 2.3. This is an extension of the case where methods have their code covered, even though they have no tests associated with them. Unsurprisingly, we found 4890 methods out of 9523 belonging to this category, because many methods delegate some work to others.

3. Methods of classes with unit tests and non-zero code coverage. This category, comprises methods that not only have a non-zero code coverage percentage (according to $\mathrm{JaCoCo}$ ), but also belong to a class with unit-test code. There are 1152 methods belonging to this category (see Section 2.5). These two conditions provide the greatest assurance that a method is indeed covered by a test. In practice, we found two cases:

(1) methods having non-zero code coverage and a test class but no unit test for the specific method, and

(2) methods having non-zero code coverage and a test class with a unit test, which can fully or partially cover the specific method.

An example of the first case can be found in method delete $(\hookleftarrow$ intupdateFlags, IProgressMonitormonitor) of the class or g.eclipse.core. internal . resources. Resource and the corresponding unit test class org.eclipse.core.tests. resource $\mathrm{s}$. ResourceTest. The method delete is executed by the method ensureDoesNotExistInWorkspace of the test class, but there is no test for checking the specific method.



Listing 2: Code of partially covered method

A representative example of the second case is the fully covered method getAllSupertypes 0 of the class org.eclipse.jdt. internal.core.hierarchy. TypeHierarchy. The corresponding test class org.eclipse.jdt.core. tests.model.TypeHierar chy contains the method testGetAllSupertypes, which tests the method getAllsupertypes 0 .

An example of a partially covered method is the method findC ontentViewerDescriptor of the class org. eclipse.compare . internal . CompareUIPlugin which is covered by $50 \%$ on instruction level and $35 \%$ by branch coverage. The corresponding test class is the org. eclipse. compare. tests. CompareUIPluginTest and it contains the methods testFindContentViewerDescriptor_ $\hookleftarrow$ UnknownType, testFindContentViewerDescriptor_TextTyp e_NotStreamAccessor, testFindContentViewerDescriptor ForTextType_StreamAccessor, which tests the method findC ontentViewerDescriptor providing different inputs.

An excerpt of findContentViewerDescriptor in Listing 2 depicts multiple if statements. According to JaCoCo, this method consists of 62 branches of which only the 23 are covered. On line 4 we can see for example the TextType and on line 3 the StreamAcce ssor condition, which seems to be related to test testFindCont entViewerDescriptorForTextType_StreamAccessor(Listing 3).

4. Non-faulty methods in incident stack traces. We noticed that there are numerous methods that appear in many incident stack traces, although they have unit tests associated with them and appear to be correct. This raises the question of how could a method appearing in so many incident reports not have been noticed and fixed by the developers.

One answer is that many of those methods are used for either debugging (such as reporting exceptions, log specific messages, handle assertions, check if something exists or is null) or for triggering code (such as run(), invoke(), execute()) and are therefore not directly associated a fault. 


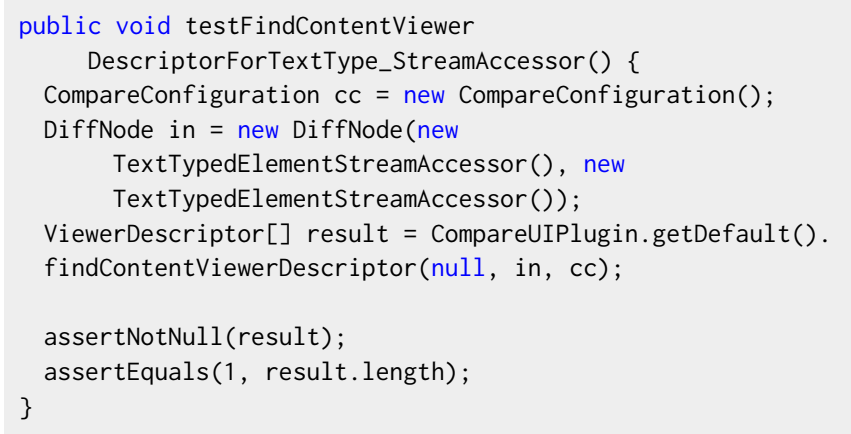

Listing 3: Test class of partially covered method



\section{Listing 4: Faulty method's call chain code}

The debugging methods are usually found at the first stack frame of the stack trace, and the triggering methods within the top- 6 and top-10 frames. For example method run(IWorkspaceRunnab leaction, IProgressMoni tormonitor) of class org.eclipse .core. internal. resources. Workspace appeared in 4293 stack traces (such as incident_360023.json) at the top-6 frames and method checkExists of class org.eclipse.core.internal. resources. Resource appeared in 2020 stack traces (such as in cident_1908559.json) at the top frame.

In addition, there are methods further from the top stack frame that just happen to be included in the stack trace as part of a faulty method's call chain, which also includes the faulty method that generated the stack trace. For example, in Listing 5, which illustrated the stack trace corresponding to the code in Listing 4, the method parent only calls method checkRemoved, but stills appears in the incident stack trace, although it is not the faulty method.

Method checkRemoved is at the top of the stack, because that is where the application generated the stack trace. The run () method is at the bottom of the stack, because this is how the program started. 2020-03-03 02:22. Page 7 of 1-12.

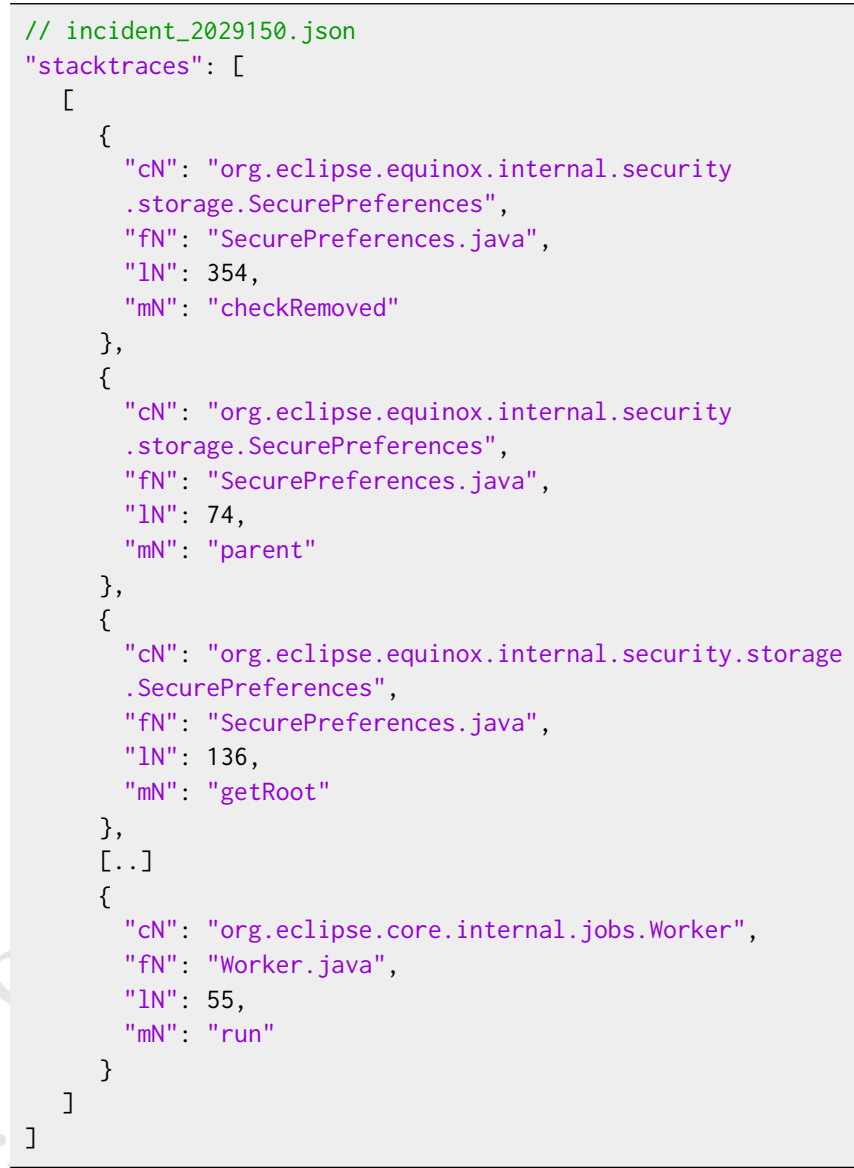

Listing 5: Faulty method's call chain

When the program started, the Java runtime executed the run() method. The run() method called getRoot and getRoot called parent, which called checkRemoved. Finally, checkRemoved threw IllegalStateException, which generated the stack trace.

\subsection{Statistical Analysis and Methods}

A typical Java stack trace is a list of the method calls or stack frames that the application was in the middle of before an error or exception was thrown (or generated manually). A stack trace can range from a single stack frame (e.g stack trace of file incident_627736.json) to 1024 frames (e.g stack trace of file incident_1655649.json), with average length of 25 frames. The position of faulty methods in a stack trace can be found in one of the top-10 stack frames according to Schroter and his colleagues [34], which mean that one of the last 10 methods that were called are likely to contain the defect.

As described in Section 2.6, there are many methods appearing at top- 6 and top- 10 positions (excluding the topmost position) that are not related to the crash. Along with the fact that the exception occurred in the first stack frame, we consider the first (topmost) frame in the stack as the method that caused the crash, and thus we decided to strictly define a method as associated with the crash when it appears in the stack trace's top position. 



Figure 3: Relationship between strictly tested and strictly crashed methods. The pie areas correspond to the colored areas of the Venn diagram.

Table 2: Crashes of Tested Methods

\begin{tabular}{lrrrrrr}
\hline & \multicolumn{4}{c}{ Unit tested } & \multicolumn{2}{c}{ Total } \\
Crashed & \multicolumn{2}{c}{ No } & \multicolumn{2}{c}{ Yes } & \multicolumn{2}{c}{} \\
\hline No & 7835 & $(82.3 \%)$ & 522 & $(5.5 \%)$ & 8357 & $(87.8 \%)$ \\
Yes & 1099 & $(11.5 \%)$ & 67 & $(0.7 \%)$ & 1166 & $(12.2 \%)$ \\
\hline Total & 8934 & $(93.8 \%)$ & 589 & $(6.2 \%)$ & 9523 &
\end{tabular}

In addition, on our preliminary qualitative analysis, we considered a method as unit-tested in one of the following cases:

(1) Method belongs to a unit-tested class but has no JaCoCo coverage data.

(2) Method does not belong to a unit-tested class but has JaCoCo coverage data.

(3) Method belongs to a unit-tested class and has JaCoCo coverage data.

We decided to consider a method as unit tested by keeping only the methods that belong to a unit-tested class and have JaCoCo line code coverage more than the median of the non-zero covered lines percentages, namely $98.3 \%$.

Based on these two definitions we generate a $2 \times 2$ contingency table containing the multivariate frequency distribution of the two variables: tested, crashed. We can then test for statistical significance (deviation from the null hypothesis of RQ1) by applying Fisher's exact test for count data $[9,10]$, which is available on the $\mathrm{R}$ statistical analysis environment $[15,32]$ as fisher. test. As we are only interested on whether testing is associated with fewer crashes (and not the reverse of whether fewer crashes are associated with testing) we test the alternative hypothesis in the less direction.

\section{RESULTS}

Here we answer our two research questions by means of statistical (RQ1) and qualitative (RQ2) analysis.

\subsection{Statistical Analysis}

To answer RQ1 regarding the association between unit tests and crashes we classified the 9523 methods as unit tested and crashed according to the criteria we specified in Section 2.7. This resulted in their categorization depicted in Figure 3 and and summarized in Table 2 .

Our question is whether testing a piece of code is associated with a lower chance of it crashing. Applying Fisher's exact test for count data, results in a $p$-value of 0.278 and an odds ratio based

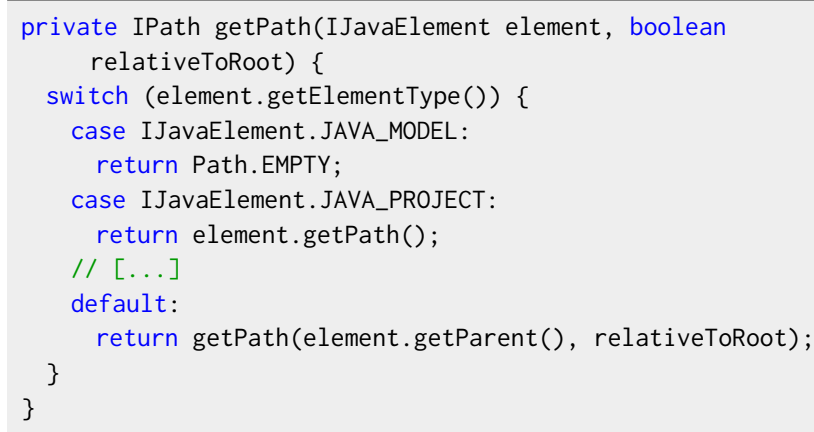

Listing 6: Example of a faulty method caused by a developer error regarding an invalid argument

on the conditional maximum likelihood estimate of 0.915 in a $95 \%$ confidence interval of $0-1.146$. Consequently, we cannot reject the null hypothesis, and conclude that our data set does not provide statistical evidence supporting the hypothesis that the presence of unit tests is associated with fewer crash incidents.

\subsection{Qualitative Analysis}

To answer RQ2 on why do unit-tested methods still fail, we analyzed the 67 methods that are strictly unit-tested and crashed. This may sound like a small number, but those methods appeared in 10608 stack traces.

By examining the stack traces and their relevant methods in the Eclipse source code, we classified crashes of unit tested methods into three categories.

1. The method contains a developer-introduced fault. These faults stem from programmer errors, such as algorithmic, logic, ordering, dependency, or consistency errors [23]. They mainly involve code parts that are missing error-handling mechanisms for code that can potentially throw exceptions, thus causing the application to crash with an uncaught exception error.

An example of a method belonging to this category, is getPath of class org. eclipse. jdt. internal . core. search. JavaSearch Scope. java (Listing 6). This was found to be the topmost method in 12 stack traces, such as incident_69854.json. The stack trace indicated that the crash occurred on line 2 which makes sense, because a NullPointerException can be thrown at that point if element is null.

This method belongs to the category 3.1 we presented in Section 2.6. It has been called by multiple other tested methods providing different element input, without testing it with a null argument. If developers had written a test that specifically checked this method, they might have covered this case.

Another example is the method consumeEmptyStatement (Listing 7). This was found to be the topmost method in 11 stack traces such as incident_1324714.json) of class org.eclipse.jdt. internal.compiler. parser.Parser. It seems to cause an In dexOutOfBoundsException on line 3.

2. The method intentionally raises an exception. There are methods that intentionally lead to crashes due to internal errors, wrong configuration settings, or unanticipated user behavior, rather than 


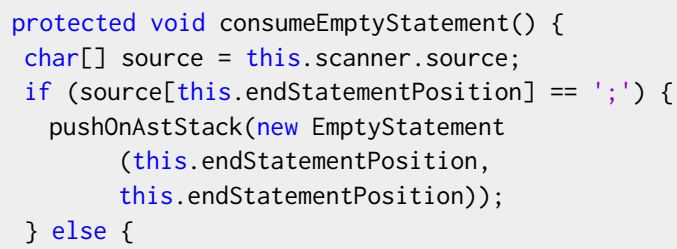

Listing 7: Example of a faulty method caused by a developer error regarding array indexing

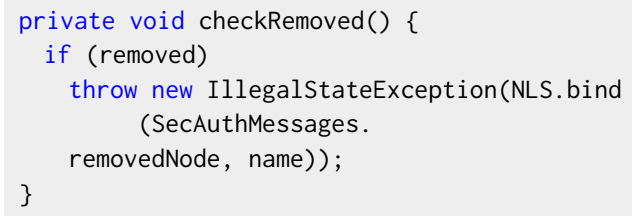

Listing 8: Example method of an internal error

private void fail(String message) throws TemplateException \{ fErrorMessage $=$ message;

throw new TemplateException(message); \}

\section{Listing 9: Example of a non-faulty method}

faults introduced through a developer oversight. Developers understood that these failures could potentially happen under unforeseen circumstances or in ways that could not be appropriately handled. As a backstop measure they intentionally throw exceptions with appropriate messages in order to log the failure and collect data that might help them to correct it in the future.

We found for example this to be the case in stack trace incid ent_2029150.json in which the first frame contains the method checkRemoved (Figure 8) of class org. eclipse.equinox. inte rnal.security.storage. SecurePreferences, which throws an IllegalStateException and logs the message "Preference node '\$node' " has been removed".

3. The method is not faulty. There are methods in the topmost stack position that simply report a failure associated with a fault in another method. These methods are the non-faulty (debugging) methods we described in category 4 of Section 2.6. An example of such a case is method fail of the class org.eclipse.jface. $t \hookleftarrow$ ext. templates. TemplateTranslator (Figure 9).

Having analyzed the crashes, we worked on understanding why those crashes occurred while there was (apparently) unit tested code. Unit testing would not help alleviate cases 2 and 3, and therefore we did not investigate further. On the other hand, methods belonging to the first case are much more interesting, so we dug deeper to understand the types of faults, failures, and their relationship to unit testing, and categorized them into the following areas.

1. a Method is not called by the class's tests. The method's test class does not call the specific method in any of the tests. The method may have been incidentally called by tests of other classes. We found for example this to be the case in method resetProcessChangeSt ate of class org. eclipse. text. undo. DocumentUndoManager.

1.b Method is not tested by the class's tests. The method's test class calls this method to setup or validate other tests, but does not 2020-03-03 02:22. Page 9 of 1-12. explicitly test the given method. We found for example this to be the case in method getPath (Listing 6) we previously saw on this section on paragraph 1 . Similarly, there are many such tests in test class org. eclipse.jdt. core. tests. compiler.parser.Pars erTest or org.eclipse.core.tests.resources. ResourceT est.

1.c Specific case is not tested. The method has a unit test, but some specific cases are not tested. Ideally, all cases should be tested to ensure that the discrete unit of functionality performs as specified under all circumstances. An example of this case is the partially (90\%) covered method iterate of the class org.eclips e.core. internal. watson. ElementTreeIterator with unit test class org. eclipse.core.tests. internal.watson.ElementT reeIteratorTest. This method causes an IndexOutofbounds Exception, but the test method does not test this case.

\section{DISCUSSION}

In isolation and at first glance, the results we obtained are startling. It seems that unit tested code is not significantly less likely to be involved in crashes. However, one should keep in mind that absence of evidence is not evidence of absence. We have definitely not shown that unit tests fail to reduce crashes.

Regarding the result, we should remember that our data come from a production-quality widely used version of Eclipse. It is possible and quite likely that the numerous faults resulting in failures were found through the unit tests we tallied in earlier development, alpha-testing, beta-testing, and production releases. As a result, the tests served their purpose by the time the particular version got released, eliminating faults whose failures do not appear in our data set.

Building on this, we must appreciate that not all methods are unit tested and not all methods are unit tested with the same thoroughness. Figure 1 shows that fewer than half of the methods and lines are unit tested. Furthermore, Figure 2 shows that code coverage within a method's body also varies a lot. This may mean that developers selectively apply unit testing mostly in areas of the code where they believe it is required.

Consequently, an explanation for our results can be that unit tests are preferentially added in complex and fault-prone code in order to weed out implementation bugs. Due to its complexity, such code is likely to contain further undetected faults, which are are in turn likely to be involved in field failures manifesting themselves as reported crashes.

One may still wonder how can unit-tested methods with a $100 \%$ code coverage be involved in crashes. Apart from the reasons we identified in Section 3.2, one must appreciate that test coverage is a complex and elusive concept. Test coverage metrics involve statements, decision-to-decision paths (predicate outcomes), predicateto-predicate outcomes, loops, dependent path pairs, multiple conditions, loop repetitions, and execution paths [21, pp. 142-145], [4]. In contrast, JaCoCo analyses coverage at the level of instructions, lines, and branches. While this functionality is impressive by industry standards, predicate outcome coverage can catch only about $85 \%$ of revealed faults [21, p. 143]. It is therefore not surprising that failures still occur in unit tested code. 
An important factor associated with our results is that failures manifested themselves exclusively through exceptions. Given that we examined failure incidents through Java stack traces, the fault reporting mechanism is unhandled Java exceptions. By the definition of an unhandled exception stack trace, all methods appearing in our data set passed an exception through them without handling it internally. This is important, for two reasons. First, unit tests rarely examine a method's exception processing; they typically do so only when the method under test is explicitly raising or handling exceptions. Second, most test coverage analysis tools fail to report coverage of exception handling, which offers an additional, inconspicuous, branching path.

It would be imprudent to use our findings as an excuse to avoid unit testing. Instead, practitioners should note that unit testing on its own is not enough to guarantee a high level of software reliability In addition, tool builders can improve test coverage analysis systems to examine and report exception handling. Finally, researchers can further build on our results to recommend efficient testing methods that can catch the faults that appeared in unit tested code and test coverage analysis processes to pinpoint corresponding risks.

\section{THREATS TO VALIDITY}

Regarding external validity, the generalizability of our findings is threatened by our choice of the analyzed project. Although Eclipse is a very large and sophisticated project, serving many different application areas, we cannot claim that our choice represents adequately all software development. For example, our findings may not be applicable to small software projects, projects in other application domains, software written in other programming languages, or multi-language projects. Finally, we cannot exclude the possibility that the selection of a specific Eclipse product and release may have biased our results. If anything, we believe additional research should look at failures associated with less mature releases.

Regarding internal validity we see four potential problems. First, the code coverage metrics we employed have room for improvement, by incorporating e.g. branch coverage or mutation testing data. Second, employing JaCoCo on an old release which may have some deprecated code and archived repos, caused some unit test failures, resulting in a lower code coverage. Third, we excluded from the JaCoCo report non-Java code that is processor architecture specific (e.g the org. eclipse.core.filesystem. linux. x86 bundle). Fourth, noise in some meaningless stack frames appearing in our stack trace dataset may have biased the results.

\section{RELATED WORK}

Among past studies researching the relationship between unit test coverage and software defects, the most related to our work are the ones that examine actual software faults. Surprisingly, these studies do not reach a widespread agreement when it comes to the relationship between the two. More specifically existing findings diverge regarding the hypothesis that a high test coverage leads to fewer defects. Mockus et al. [28], who studied two different industrial software products, agreed with the hypothesis and concluded that code coverage has a negative correlation with the number of defects. On the other hand, Gren and Antinyan's work [13] suggests that unit testing coverage is not related to fewer defects and there is no strong relationship between unit testing and code quality. A more recent study by the same primary author [2], investigated an industrial software product, and also found a negligible decrease in defects when coverage increases, concluding that test unit coverage is not a useful metric for test effectiveness.

Furthermore, in a study of seven Java open source projects, Petric et al. found that the majority of methods with defects had not been covered by unit tests [31], deducing that the absence of unit tests is risky and can lead to failures. On the other hand, Kochhar et al. in another study of one hundred Java projects, did not find a significant correlation between code coverage and defects [24].

The above mentioned studies cover only fixed faults. In our research, we work with stack traces, which enable us to analyze field-reported failures associated with crashes. The associated faults include those that have not been fixed, but exclude other faults that are not associated with crashes, such as divergence from the expected functionality or program freezes. Furthermore, through the crash reports we were unable to know the faulty method associated with the crash. However, by placing our matched crash methods in three groups according to their respective position in the stack trace (in the very first stack frame, within the top- 6 and the top-10 stack frames) we could obtain useful bounds backed by empirical evidence [34] regarding the coverage of methods that were likely to be defective.

Considerable research associating testing with defects has been performed on the relationship between test-driven development and software defects. Test-driven development (TDD) is centered around rapid iterations of writing test cases for specifications and then the corresponding code [5]. As a practice it obviously entails more than implementing unit tests, but absence of evidence of TDD benefits should also translate to corresponding absence of benefits through simple unit testing, though the benefits of TDD will not necessarily translate into benefits of unit testing. In a review of the industryâĂŹs and academiaâĂŹs empirical studies, MÃdkinen and MÃijnch [26] found that TDD has positive effects in the reduction of defects a result also mirrored in an earlier meta-analysis [33] and a contemporary viewpoint [29]. In industry, an IBM case study found that test-driven development led to $40 \%$ fewer defects [38]. In academia, classroom experiment results showed that students produce code with $45 \%$ fewer defects using TDD [8]. On the other hand, experimental results by Wikerson and Mercer failed to show significant positive effects [37].

The study by Jia and Harman [19] shows clear evidence that mutation testing has gained a lot popularity during the past years. The majority of researchers concluded that high mutation score improves fault detection [30]. Furthermore, mutation testing can reveal additional defect cases beyond real faults [1]. However, mutants can only be considered substitute of real faults under specific circumstances [22].

Apart from Schroter and his colleagues [34], a number of researchers have studied the Eclipse IDE and most of them have focused on predicting defects. Most notably, Zimmermann and his colleagues provided a dataset mapping defects from the Eclipse database to specific source code locations annotated with common complexity metrics [41], while Zhang [40] based on Eclipse data, yet again, suggested lines of code as a simple but good predictor of defects. 


\section{CONCLUSIONS}

Software testing contributes to code quality assurance and helps developers detect and correct program defects and prevent failures. Being an important and expensive software process activity it has to be efficient. In our empirical study on the Eclipse project we used the JaCoCo tool and a class source code matching procedure to measure the test coverage, and we analyzed field failure stack traces to assess the effectiveness of testing. Our results indicate that unit testing on its own may not be a sufficient method for preventing program failures. Many methods that were covered by unit tests were involved in crashes, which may mean that the corresponding unit tests were not sufficient for uncovering the corresponding faults. However, it is worth keeping in mind that failures manifested themselves through exceptions whose branch coverage JaCoCo is not reporting. Research building on ours can profitably study the faults that led to the failures we examined in order to propose how unit testing can be improved to uncover them, and how test coverage analysis can be extended to suggest these tests.

\section{ACKNOWLEDGMENTS}

We thank Philippe Krief and Boris Baldassari for their invaluable help regarding the Eclipse incident data set. Panos Louridas provided insightful comments on an earlier version of this manuscript. Dimitris Karlis expertly advised us on the employed statistical methods. This work has been partially funded by: the CROSSMINER project, which has received funding from the European Union Horizon 2020 research and innovation programme under grant agreement No 732223; the FASTEN project, which has received funding from the European Union Horizon 2020 research and innovation programme under grant agreement No 82532; the GSRT 2018 Research Support grant 11312201; and the Athens University of Economics and Business Research Centre Original Scientific Publications 2019 grant EP-3074-01.

\section{REFERENCES}

[1] James H Andrews, Lionel C Briand, and Yvan Labiche. 2005. Is Mutation an Appropriate Tool for Testing Experiments?. In Proceedings of the 27th international conference on Software engineering. ACM, 402-411.

[2] V. Antinyan, J. Derehag, A. Sandberg, and M. Staron. 2018. Mythical Unit Test Coverage. IEEE Software 35, 3 (May 2018), 73-79. https://doi.org/10.1109/MS 2017.3281318

[3] Boris Baldassari. 2018. StackTraces - Incidents. Available online https://softwaredata.org/datasets/aeri-stacktraces/resources/incidents_analysis.pdf. Accessed January 16,2020

[4] Thomas Ball, Peter Mataga, and Mooly Sagiv. 1998. Edge Profiling Versus Path Profiling: The Showdown. In Proceedings of the 25th ACM SIGPLAN-SIGACT Symposium on Principles of Programming Languages (POPL '98). ACM, New York, NY, USA, 134-148. https://doi.org/10.1145/268946.268958

[5] Kent Beck. 2003. Test-Driven Development: By Example. Addison-Wesley, Boston.

[6] Kent Beck and Erich Gamma. 1998. Test Infected: Programmers Love Writing Tests. Fava Report 3, 7 (July 1998), 37-50.

[7] A. Causevic, D. Sundmark, and S. Punnekkat. 2011. Factors Limiting Industrial Adoption of Test Driven Development: A Systematic Review. In Fourth IEEE International Conference on Software Testing, Verification and Validation. 337-346. https://doi.org/10.1109/ICST.2011.19

[8] Stephen H Edwards. 2003. Using Test-Driven Development in the Classroom Providing Students with Automatic, Concrete Feedback on Performance. In Proceedings of the international conference on education and information systems technologies and applications EISTA, Vol. 3. Citeseer.

[9] R. A. Fisher. 1922. On the Interpretation of x2 from Contingency Tables, and the Calculation of P. Fournal of the Royal Statistical Society 85, 1 (1922), 87-94.

[10] R. A. Fisher. 1935. The Logic of Inductive Inference. fournal of the Royal Statistical Society 98, 1 (1935), 39-82.

2020-03-03 02:22. Page 11 of 1-12
[11] David Geer. 2005. Eclipse becomes the dominant Java IDE. Computer 38, 7 (2005), $16-18$.

[12] Rahul Gopinath, Carlos Jensen, and Alex Groce. 2014. Code Coverage for Suite Evaluation by Developers. In Proceedings of the 36th International Conference on Software Engineering (ICSE 2014). ACM, New York, NY, USA, 72-82. https: //doi.org/10.1145/2568225.2568278

[13] Lucas Gren and Vard Antinyan. 2017. On the Relation Between Unit Testing and Code Quality. In 2017 43rd Euromicro Conference on Software Engineering and Advanced Applications (SEAA). IEEE, 52-56.

[14] Marc R. Hoffmann, B. Janiczak, and E. Mandrikov. 2018. JaCoCo Java Code Coverage Library. Available online https://www.eclemma.org/jacoco/. Current 2019-01-20.

[15] Ross Ihaka and Robert Gentleman. 1996. R: A Language for Data Analysis and Graphics. Journal of Computational and Graphical Statistics 5, 3 (Sept. 1996), 299-314.

[16] D.C. Ince, L. Hatton, and J. Graham-Cumming. 2012. The Case for Open Program Code. Nature 482 (February 2012), 485-488. https://doi.org/10.1038/nature10836

[17] Laura Inozemtseva and Reid Holmes. 2014. Coverage is Not Strongly Correlated with Test Suite Effectiveness. In Proceedings of the 36th International Conference on Software Engineering (ICSE 2014). ACM, New York, NY, USA, 435-445. https: //doi.org/10.1145/2568225.2568271

[18] International Organization for Standardization. 2017. Systems and software engineering - Vocabulary. ISO, Geneva, Switzerland. ISO/IEC 24765:2017.

[19] Yue Jia and Mark Harman. 2010. An Analysis and Survey of the Development of Mutation Testing. IEEE Transactions on Software Engineering 37, 5 (2010), 649-678.

[20] Y. Jia and M. Harman. 2011. An Analysis and Survey of the Development of Mutation Testing. IEEE Transactions on Software Engineering 37, 5 (Sept. 2011), 649-678. https://doi.org/10.1109/TSE.2010.62

[21] Paul C. Jorgensen. 2002. Software Testing: A Craftsman's Approach. CRC Press, Boca Raton, FL

[22] René Just, Darioush Jalali, Laura Inozemtseva, Michael D Ernst, Reid Holmes, and Gordon Fraser. 2014. Are Mutants a Valid Substitute for Real Faults in Software Testing?. In Proceedings of the 22nd ACM SIGSOFT International Symposium on Foundations of Software Engineering. ACM, 654-665.

[23] Andrew J Ko and Brad A Myers. 2005. A framework and methodology for studying the causes of software errors in programming systems. Fournal of Visual Languages \& Computing 16, 1-2 (2005), 41-84.

[24] Pavneet Singh Kochhar, David Lo, Julia Lawall, and Nachiappan Nagappan. 2017. Code Coverage and Postrelease Defects: A Large-Scale Study on Open Source Projects. IEEE Transactions on Reliability 66, 4 (2017), 1213-1228.

[25] Pavneet Singh Kochhar, Ferdian Thung, and David Lo. 2015. Code Coverage and Test Suite Effectiveness: Empirical Study with Real Bugs in Large Systems. In Software Analysis, Evolution and Reengineering (SANER), 2015 IEEE 22nd International Conference on. IEEE, 560-564.

[26] Simo Mäkinen and Jürgen Münch. 2014. Effects of Test-Driven Development: A Comparative Analysis of Empirical Studies. In Software Quality. Model-Based Approaches for Advanced Software and Systems Engineering, Dietmar Winkler, Stefan Biffl, and Johannes Bergsmann (Eds.). Springer International Publishing, Cham, 155-169.

[27] T. J. McCabe. 1976. A Complexity Measure. IEEE Transactions on Software Engineering 2, 4 (1976), 308-320. https://doi.org/10.1109/tse.1976.233837

[28] Audris Mockus, Nachiappan Nagappan, and Trung T Dinh-Trong. 2009. Test Coverage and Post-Verification Defects: A Multiple Case Study. In Empirical Software Engineering and Measurement, 2009. ESEM 2009. 3rd International Symposium on. IEEE, 291-301.

[29] Hussan Munir, Misagh Moayyed, and Kai Petersen. 2014. Considering Rigor and Relevance when Evaluating Test Driven Development: A systematic review. Information and Software Technology 56, 4 (2014), 375-394.

[30] Mike Papadakis, Donghwan Shin, Shin Yoo, and Doo-Hwan Bae. 2018. Are Mutation Scores Correlated with Real Fault Detection? A Large Scale Empirical Study on the Relationship Between Mutants and Real Faults. In 2018 IEEE/ACM 40th International Conference on Software Engineering (ICSE). IEEE, 537-548.

[31] Jean Petrić, Tracy Hall, and David Bowes. 2018. How Effectively Is Defective Code Actually Tested?: An Analysis of JUnit Tests in Seven Open Source Systems. In Proceedings of the 14th International Conference on Predictive Models and Data Analytics in Software Engineering. ACM, 42-51.

[32] R Core Team. 2012. R: A Language and Environment for Statistical Computing. (2012)

[33] Yahya Rafique and Vojislav B Mišić. 2012. The Effects of test-Driven Development on External Quality and Productivity: A Meta-Analysis. IEEE Transactions on Software Engineering 39, 6 (2012), 835-856.

[34] Adrian Schroter, Adrian Schröter, Nicolas Bettenburg, and Rahul Premraj. 2010. Do Stack Traces Help Developers Fix Bugs?. In 2010 7th IEEE Working Conference on Mining Software Repositories (MSR 2010). IEEE, 118-121.

[35] Keith Stobie. 2005. Too Darned Big to Test. Queue 3, 1 (Feb. 2005), 30 âĂȘ37. https://doi.org/10.1145/1046931.1046944 
[36] Mustafa M. Tikir and Jeffrey K. Hollingsworth. 2002. Efficient Instrumentation for Code Coverage Testing. In Proceedings of the 2002 ACM SIGSOFT International Symposium on Software Testing and Analysis (ISSTA '02). ACM, New York, NY, USA, 86-96. https://doi.org/10.1145/566172.566186

[37] Jerod W Wilkerson, Jay F Nunamaker, and Rick Mercer. 2011. Comparing the Defect Reduction Benefits of Code Inspection and Test-Driven Development. IEEE Transactions on Software Engineering 38, 3 (2011), 547-560.

[38] Laurie Williams, E Michael Maximilien, and Mladen Vouk. 2003. Test-Driven Development as a Defect-Reduction Practice. In 14th International Symposium on Software Reliability Engineering, 2003. ISSRE 2003. IEEE, 34-45.
[39] T. W. Williams, M. R. Mercer, J. P. Mucha, and R. Kapur. 2001. Code Coverage, What Does it Mean in Terms of Quality? In Annual Reliability and Maintainability Symposium. 2001 Proceedings. International Symposium on Product Quality and Integrity. 420-424. https://doi.org/10.1109/RAMS.2001.902502

[40] Hongyu Zhang. 2009. An Investigation of the Relationships Between Lines of Code and Defects. In 2009 IEEE International Conference on Software Maintenance. IEEE, 274-283.

[41] Thomas Zimmermann, Rahul Premraj, and Andreas Zeller. 2007. Predicting Defects for Eclipse. In Third International Workshop on Predictor Models in Software Engineering (PROMISE'07: ICSE Workshops 2007). IEEE, 9-9. 